Proc. Estonian Acad. Sci. Eng., 2005, 11, 2, 106-125

\title{
Physics of inertial microparticles transfer in turbulent boundary layers
}

\begin{abstract}
Alfredo Soldati
Joint Department of Fluid Dynamics, Hydraulics and Energy Technology, University of Udine, Via delle Scienze 208, 33100 Udine, Italy; soldati@uniud.it

Received 22 March 2004

Abstract. Turbulent dispersed flows in boundary layers are crucial in a number of industrial and environmental applications. In most applications, the key information is particle spatial distribution and it is known that inertial particles in turbulent flows distribute preferentially avoiding strong vortical regions and segregating into straining regions. It is also known that, in the specific case of boundary layers, this preferential accumulation occurs along the wall in a macroscopic way. Numerical and experimental works show that fluid motions in turbulent boundary layers are intermittent and have a strongly organized and coherent nature, represented by the large scale motions. These motions, even though not exactly repeatable and only quasideterministic, control the transport of the dispersed species. In this work, direct numerical simulations of turbulence in upward boundary layers in pipes and channels and Lagrangian tracking of inertial particles are employed to examine particle transfer in connection with the dynamics of the flow structures populating the boundary layer. It is argued that the local syncronicity between the events in the turbulence regeneration cycle and the mechanisms, controlling particle fluxes toward and away from the wall, determine the macroscopic net particle fluxes.
\end{abstract}

Key words: particles, turbulence, boundary layer, coherent structures.

\section{INTRODUCTION AND BACKGROUND}

A relevant feature of turbulence fields is the dynamics of large-scale coherent structures $\left[{ }^{1-3}\right]$. When inertial particles are dispersed in the field, these structures generate preferentially directed non-random motions of particles, leading to nonuniform concentration and to long-term accumulation $\left[{ }^{4-6}\right]$. In the specific case of turbulent boundary layer, the local interaction between particles and turbulence structures will lead to a remarkably macroscopic behaviour, producing particle accumulation in the viscous sublayer $\left[{ }^{7-11}\right]$. 
Particle transfer in the wall region of a turbulent boundary layer is a highly non-uniform and intermittent phenomenon which depends on the local dynamics of turbulence structures. Decades of extensive studies have clarified several issues concerning the correlation between turbulence structures and particle dynamics $\left[{ }^{9,12-16}\right]$. To resume several of those, we refer to our previous works $[9,10,17]$ in which the causal relationship between turbulence structures and particle transfer mechanisms is addressed.

Consider first the case of upward channel flow examined in $\left[{ }^{9}\right]$. A representative view of particle dynamics and distribution in a turbulent boundary layer is provided in Fig. 1a, where a cross-sectional view in the half domain of the channel at the dimensionless simulation time $t^{+}=2700$ is shown. The superscript "+" identifies wall units, i.e. variables made dimensionless by using viscosity, $\nu$, and friction velocity $u_{\tau}=\left(\tau_{w} / \rho\right)^{\frac{1}{2}}$, where $\tau_{w}$ is wall shear stress and $\rho$ is fluid density. In Fig. 1a, the flow is directed toward the reader and particles with relaxation time $\tau_{p}^{+}$ equal to 116.3 are considered. Particle relaxation time is defined as $\tau_{p}=\rho_{p} d_{p}^{2} / 18 \mu$, where $\rho_{p}, d_{p}$ and $\mu$ are particle density, particle diameter and fluid dynamic viscosity, respectively. Figure 1a shows a number of features which can classify the process of particle dispersion and transfer in turbulent boundary layer. First, we can observe that particles are not homogeneously distributed along the channel but they tend to cluster, particularly around the large vortical structures. From these clusters, particles are transported toward the wall, where they accumulate in specific "reservoirs" (one of these is indicated by the black circle) where concentration build-up occurs. These accumulation regions are classified by flow streamwise velocity lower than the mean $\left[{ }^{13,15}\right]$. Particles tend to stay longer in these lowspeed regions so that eventually particle concentration increases near the wall. To quantify the near-wall accumulation, the particle number density distribution is plotted as a function of the non-dimensional distance $z^{+}$from the wall in Fig. 1b. A logarithmic scale is used to capture the details of particle behaviour in the proximity of the wall. The concentration profile is developing with time and, at the instant

(a)

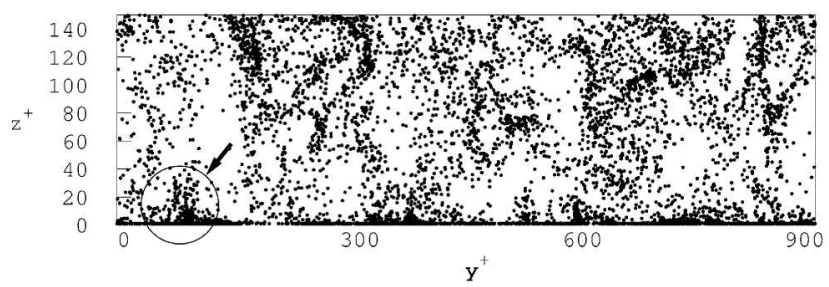

(b)

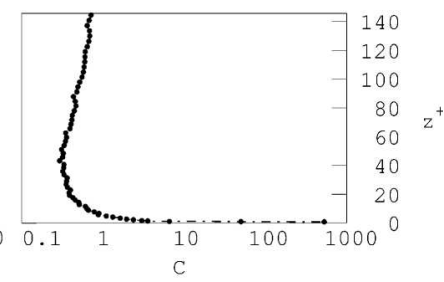

Fig. 1. Instantaneous distribution of $\tau_{p}^{+}=111.6$ particles at time $t^{+}=2700$ in vertical upward channel flow: (a) view of particle position in the $y z$ plane for $700<x^{+}<1000$; (b) corresponding $y z$ plane average number density distribution as a function of the coordinate of the wall normal. 
captured in Fig. 1b, we observe that particle number density profile has reached a maximum in the near-wall region $\left(0<z^{+}<20\right)$. This behaviour can be viewed as the consequence of the turbulence non-homogeneity [ $\left.{ }^{5,17}\right]$ and has been observed in a number of previous works $\left[{ }^{8,10,11}\right]$.

Particle wall transfer mechanisms are similar also in different flow geometries $\left[{ }^{18,19}\right]$. Referring to our previous works $\left[{ }^{10,20}\right]$, in which we simulated an upward flow in a pipe at $R e_{\tau}=337$, we observe similar features in Fig. 2. In this figure the instantaneous top view of particle distribution for three different particle sets, each characterized by a different relaxation time $\left(\tau_{p}^{+}=3.2,27.9\right.$ and 111.6), is shown at different times of the simulation. For clarity of presentation, we show only a

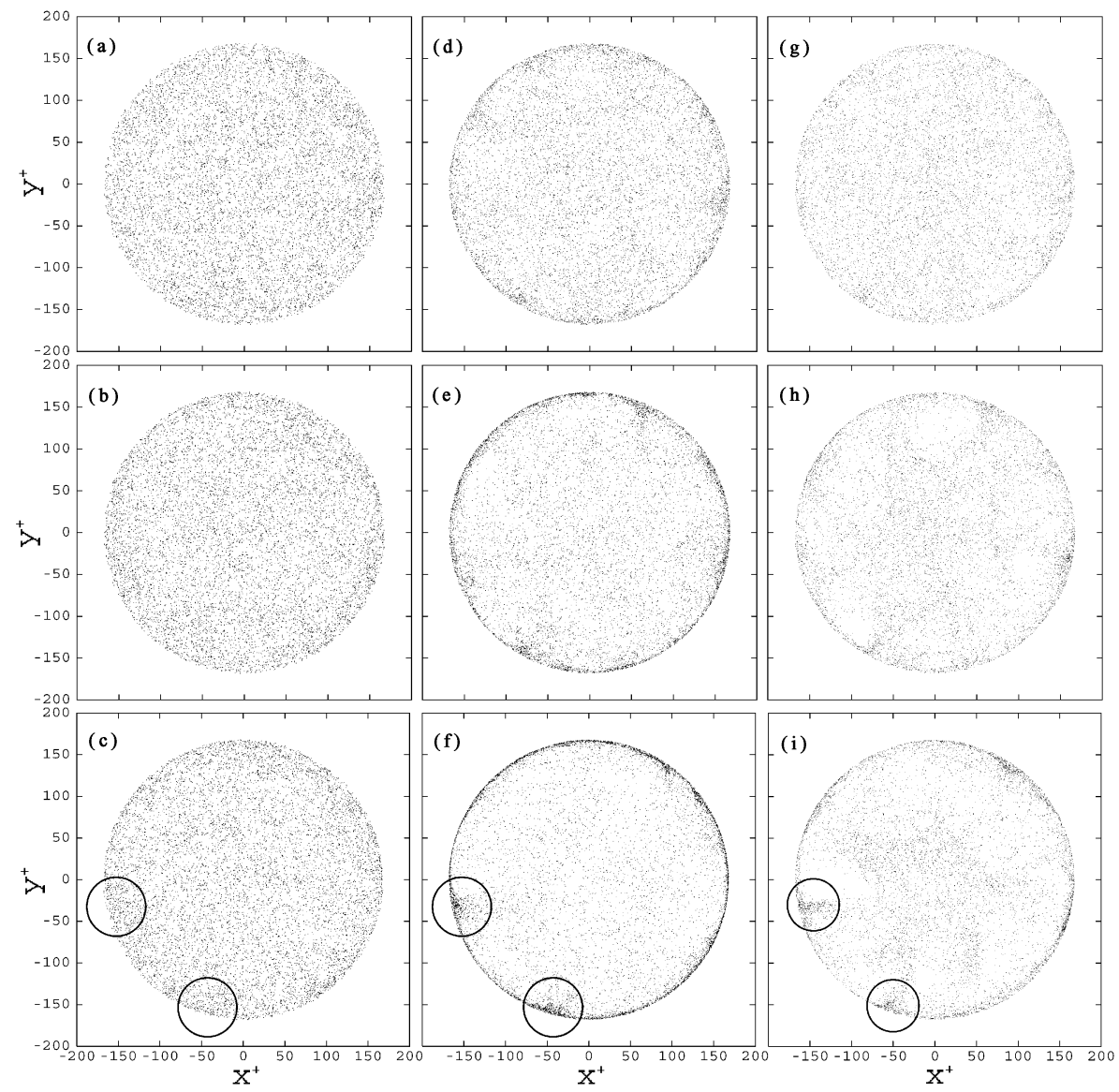

Fig. 2. Top view of the particle distribution in a pipe flow at different simulation times: (a), (d) and (g) - distribution at time $t^{+}=174$ for $\tau_{p}^{+}=3.2, \tau_{p}^{+}=27.9, \tau_{p}^{+}=111.6$, respectively; (b), (e) and (h) - distribution at time $t^{+}=695$ for $\tau_{p}^{+}=3.2, \tau_{p}^{+}=27.9, \tau_{p}^{+}=111.6$, respectively; (c), (f) and (i) - distribution at time $t^{+}=1150$ for $\tau_{p}^{+}=3.2, \tau_{p}^{+}=27.9$, $\tau_{p}^{+}=111.6$, respectively. 
pipe length of about 300 wall units before the outlet. At time $t^{+}=0$, particles are uniformly distributed in the computational domain.

From Fig. 2, a, b and c, it is apparent that the distribution of $\tau_{p}^{+}=3.2$ particles hardly changes during the initial transient of about $1000 t^{+}$: due to their small relaxation time, these particles behave approximately as fluid tracers and need longer times to modify their initial uniform distribution.

A different behaviour is found for both $\tau_{p}^{+}=27.9$ and 111.6 particles. At $t^{+}=695$ (Fig. 2, e and h), the near-wall build-up of particles develops. This behaviour has been reported in several previous works $\left[{ }^{18,21,22}\right]$. Figure 2, $\mathrm{f}$ and $\mathrm{i}$, taken at $t^{+}=1150$, shows more clearly the non-random fashion of particle clustering. In particular, the black circles indicate particle clusters that approach the walls through preferential avenues and strike the wall. Focusing on Fig. 2i, we observe large void regions, indicating the presence of a large streamwise vortex. Due to the small Reynolds number of the flow, these void regions can extend up to more than 80 wall units from the wall, reaching roughly half of the cylinder radius.

It is apparent that particle transfer processes are dominated by the dynamics of turbulent structures in the proximity of the wall. The object of this work is to describe the complex causal relationship between the dynamics of turbulence structures near the wall, the local mechanisms of particle transfer and the overall macroscopic observation of patricle preferential distribution, elaborated in our previous papers $\left[{ }^{9,10}\right]$. We will first review the details of the dynamics of the turbulence regeneration cycle and secondly we will try to explain the phenomenology of particle transfer with preferential concentration on the dynamics of coherent structures of the turbulence.

For the numerical experiments, we will only refer to the channel flow geometry for which the methodology was discussed in $\left[{ }^{9}\right]$. Particles are introduced in a numerically simulated turbulent Poiseuille flow of air (incompressible and Newtonian) at $R e_{\tau}=u_{\tau} h / \nu=150$ which, for a channel with half-width $h=$ $4 \mathrm{~cm}$, gives an average velocity of $1.65 \mathrm{~m} \mathrm{~s}^{-1}$. The reference geometry consists of two infinite vertical flat parallel walls: periodic boundary conditions are imposed on the fluid velocity field both in streamwise $(x)$ and spanwise $(y)$ directions and no-slip boundary conditions are imposed at the walls. The computational domain is $1885 \times 942 \times 300$ wall units in $x, y$ and $z$ with $64 \times 64 \times 65$ nodes. The time step used for the fluid was $\Delta t^{+}=0.35325$ in wall time units. Since we are interested in the influence of turbulence structures on particle behaviour, we assume that particle number density and particle size are both small: the feedback of the particles onto the gas flow is negligible. As demonstrated in the experiments by Kaftori et al. $\left[{ }^{14}\right]$, turbulence characteristics change slightly for dilute dispersion, thus permitting us to obtain results of general relevance. Particles are assumed to be pointwise, rigid, spherical and their Lagrangian equation of motion includes the effects of particle inertia, Stokes drag and Saffman lift force. Results presented in the following refer to the case of upward flow (gravity opposed to flow direction), otherwise indicated. Three sets of $48^{3}$ flyash particles $\left(\tau_{p}^{+}=3.8,29.1,116.3\right)$ were initially released at random locations within the computational box. We considered that a particle is 
elastically reflected away from the wall when its centre is at a distance of less than one particle radius from the wall.

\section{TURBULENCE STRUCTURE AT THE WALL}

We consider in this section the boundary layer, which occurs in a fully developed turbulent channel flow. In a turbulent boundary layer, turbulent mixing close to the wall is controlled by the Reynolds stresses. Of the convective correlation events, which characterize the Reynolds stresses, strong local motions of fluid, which are called ejections and sweeps, are the most efficient transfer agents. Specifically, ejections bring the low-momentum fluid close to the wall into the outer region whereas sweeps bring the high-momentum fluid from the outer flow into the wall region. Ejections and sweeps control momentum transfer at the wall and are also well correlated to heat transfer $\left[{ }^{23,24}\right]$ and mass transfer $\left[{ }^{25}\right]$ at the wall. A complete characterization of sweeps and ejections and of their generation mechanisms is thus fundamental in order to gain insight into the physics of turbulence structure at the wall.

We visualize single events and the dynamics of the structures, which control their occurrence, in Fig. 3 where the footprint of the shear-stress at the wall is shown together with sweeps and ejections. In this figure, flow is from the left to the right along the $x$ direction. Sweeps and ejections are identified with the same isocontour of the instantaneous surface at $u^{\prime} w^{\prime}=-3$ in wall units. Ejections (blue lumps) correspond to low shear stress regions, shown in blue, whereas sweeps (gold lumps) correspond to high shear stress regions, shown in red. Sweeps and ejections are just the final outcome of the dynamics of turbulence structures in the wall layer, and there is still some uncertainty about the mechanisms which generate and maintain the sweep/ejection events. They appear to be generated by the quasi-streamwise vortices which populate the near-wall region. Quasi-streamwise vortices have been the object of a number of works starting with $\left[{ }^{26}\right]$. There is a general agreement about their characteristics: quasi-streamwise vortices appear to have a characteristic length of about 200 wall units and a spacing of about 400 wall units $\left[{ }^{27-30}\right]$. Quasi-streamwise vortices are slightly tilted upward, about $9^{\circ}$ in average $\left[{ }^{29}\right]$; clockwise and counterclockwise rotating vortices are also slightly tilted about $4^{\circ}$ left and right, respectively. These vortices, which are responsible for pumping fluid towards and away from the wall, may be identified by using pressure, vorticity or other indicators [ $\left.{ }^{31-33}\right]$. A convenient method for identification is based on calculating the eigenvalues of the strain rate tensor $\Omega\left[{ }^{34-36}\right]$. The vector $\Omega$ is based on the identification of flow regions where the rate-of-deformation tensor $\partial u_{i} / \partial x_{j}$ exhibits complex eigenvalues. Thus, $\boldsymbol{\Omega}$ is defined as follows:

$$
\boldsymbol{\Omega}=\operatorname{Im}\left(\lambda_{c}\right) \frac{\mathbf{e}_{\lambda_{r}}}{\left|\mathbf{e}_{\lambda_{r}}\right|} \frac{\mathbf{e}_{\lambda_{r}} \cdot\left[\operatorname{Re}\left(\mathbf{e}_{\lambda_{c}}\right) \times \operatorname{Im}\left(\mathbf{e}_{\lambda_{c}}\right)\right]}{\left|\mathbf{e}_{\lambda_{r}} \cdot\left[\operatorname{Re}\left(\mathbf{e}_{\lambda_{c}}\right) \times \operatorname{Im}\left(\mathbf{e}_{\lambda_{c}}\right)\right]\right|},
$$


where $\operatorname{Im}\left(\lambda_{c}\right)$ is the imaginary part of the pair of complex eigenvalues, $\operatorname{Re}\left(\mathbf{e}_{\lambda_{c}}\right)$ and $\operatorname{Im}\left(\mathbf{e}_{\lambda_{c}}\right)$ are the real and the imaginary part of the conjugate complex eigenvectors, corresponding to the complex eigenvalues, $\mathbf{e}_{\lambda_{r}}$ is the eigenvector, corresponding to the real eigenvalue $\lambda_{r}$. The vector $\Omega$ represents the strength and direction of the rotation of the streamlines.

We drew an isosurface plot of $\Omega$, selecting a value equal to $25 \%$ of the instantaneous maximum of $\boldsymbol{\Omega}$, which is high enough to capture only the strong vortices $\left[{ }^{37,38}\right]$. In Fig. 4, two counterrotating vortices, identified by one isosurface of $\Omega$, are shown together with the ejections and sweeps they generate. The elongated red and pale blue structures are two isosurfaces with the same absolute value of $\boldsymbol{\Omega}$ (but of opposite sign) and indicate clockwise (red) and counterclockwise (pale blue) rotating vortices. Flow is going from bottom left to top right and vortices appear tilted away from the wall by the mean strain rate. The blue lumps of fluid in between the two vortices are ejections and the green lumps of fluid outside the two vortices are sweeps. Ejections and sweeps also appear to be affected by the mean strain rate. Owing to the continuous action of the quasi-streamwise vortices in generating sweeps and ejections, regions between two vortices such as those shown in Fig. 4 are characterized by a streamwise velocity lower than the mean, whereas the regions outside the two vortices are characterized by a streamwise velocity higher than the mean. Specifically, the regions with velocity lower than the mean are called low-speed streaks, whereas the regions with velocity higher than the mean are called high-speed regions. Low-speed streaks are sinuous regions about 1000 wall units long and are more coherent than the high-speed regions. Also, low-speed streaks are long-lived wall structures which survive a number of quasi-streamwise vortex generations, the time duration of these vortices being much shorter.

As clearly shown in Fig. 5, many quasi-streamwise vortical structures overlap in $x$ as a staggered array and appear associated with one low-speed streak - a 650 wall unit long piece of one low-speed streak.

\section{TURBULENCE STRUCTURES AND PARTICLE TRANSFER MECHANISMS}

In previous papers $\left[{ }^{9,17,39}\right]$, we presented both qualitative and quantitative results to put in evidence the mechanisms by which particles are transferred to the wall by the sweeps and are eventually re-entrained into the outer flow by the ejections. We also determined how the strongly coherent Reynolds stress events influence particle fluxes. The picture is that particles enter the wall layer, advected by the strongly coherent sweeps, and exit the wall layer, advected by the strongly coherent ejections. However, exit fluxes are much weaker than inlet fluxes. We shall try to address that issue in this section. Specifically, our aim is to explain, from a physical viewpoint, the mechanisms for particle segregation within the boundary layer. To this aim, we have to link all the phenomena observed to the dynamics of the near-wall turbulent vortical structures. 
The vortical structures, which dominate the wall layer dynamics, are the aforementioned quasi-streamwise vortices, which generate sweeps on the downwash side and ejections on the upwash side. In turn, ejections contribute to the maintenance of the lifted low-speed streaks on the upwash side of the quasi-streamwise vortices. Recent results $\left[{ }^{29,38,40}\right]$ show that clockwise and counterclockwise quasi-streamwise vortices appear flanking the low-speed streak as a staggered array in most of the cases. This is shown in Fig. 5. Only rarely do clockwise and counterclockwise quasi-streamwise vortices appear together. This scenario is consistent with the mechanism proposed by Adrian et al. $\left[{ }^{41}\right]$, who identify and characterize the fundamental role of packets of hairpin vortices travelling at the same convection velocity in the turbulence regeneration mechanism.

Considering the state of the art on turbulence structures at the wall and the results reported in the previous section we observe that: i) the strongly coherent sweeps and ejections are responsible for transferring towards and away from the wall most of the particles of the size range we investigated, ii) the strongly coherent sweeps and ejections are generated by the strongly coherent near-wall quasistreamwise vortices, and iii) in low-Reynolds-number turbulent boundary layers, the strongly coherent archetype quasi-streamwise vortices have been identified and sized by several authors $\left[{ }^{8,28-30,38,40,42,43}\right]$. In most of the cases, these vortices are not paired to an equal-size parallel counter-rotating vortex. Thus, we can exploit the schematics in Fig. 6 to pose a fundamental question. With reference to Fig. 6, we can envisage the following cycle for particles, initially in the outer flow: if a particle is captured by a sweep, it moves along a curved trajectory around the quasistreamwise vortex, generating the sweep, approaches the wall and moves between the vortex and the wall. During this phase, the particle may touch the wall or not. Then, the particle is on the upwash side of the vortex and is subject to the influence of the ejection. The next step involves trespassing the lifted low-speed streak and

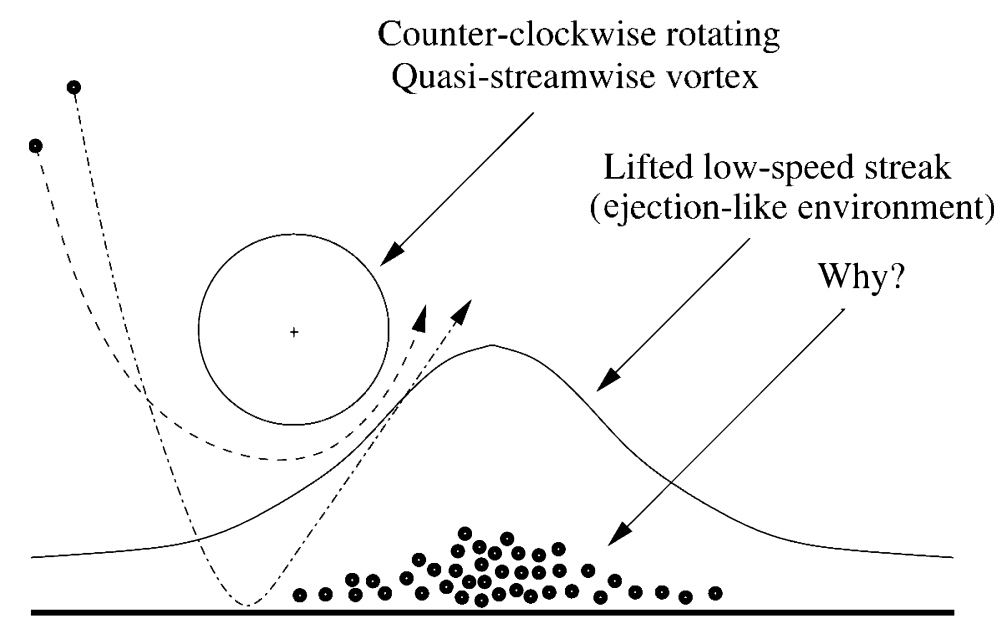

Fig. 6. Schematic mechanisms for particle transfer to the wall and away from the wall. 
exiting from the wall layer. Considering in particular the conservative conditions of our simulations, with perfectly elastic rebound, particles should migrate toward the surface of the lifted low-speed streak, which is an ejection-like environment, and find an ejection strong enough to drive them into the outer flow. Yet most of the particles remain trapped under the lifted low-speed streak.

To investigate the mechanisms, leading to particle accumulation under the lifted low-speed streaks, we examined a large number of snapshots, showing the action of quasi-streamwise vortices on particle transfer in the wall region. We show one of these in Fig. 7a, which focuses on a $y-z$ window of the computational domain, extruded for the length of one streamwise cell (dimensions are $30 \times 58 \times 108$ in dimensionless wall units) at time $t^{+}=1412$. The main character in this picture is the green counter-clockwise-rotating quasi-streamwise vortex, visualized by the streamline rotation vector $\Omega$. This vortex is characterized by positive $\omega_{x}$ vorticity, centred at $z^{+}=36$. We also show, for $\tau_{P}^{+}=3.8$, the particles with negative $w_{P}-$ directed to the wall (black circles), the particles with positive $w_{P}-$ directed away from the wall (blue circles) and the particles with $w_{P}$ almost zero, $-\left|w_{P}\right|<10^{-3}$ in wall units (empty circles). The action of the large vortex in transferring the black particles to the wall and the blue particles away from the wall is apparent. Particles with negligible wall-normal velocity accumulate under the lifted low-speed streak, which we described with a blue isosurface, indicating a streamwise velocity value of $\left.0.56 U_{C}{ }^{44}\right]$, where $U_{C}=16.76$ is the centreline velocity. The low-speed streak appears lifted by the counterclockwise quasi-streamwise vortex.

If we characterize all the coherent vortical structures present in the area with the same $|\Omega|$ isosurface but with both signs, a secondary, but relevant, character in this picture appears as a red isosurface, identifying a smaller counter-rotating quasi-streamwise vortex of negative $\omega_{x}$ vorticity, centred at $z^{+}=9$ and extending well into the viscous wall layer. The presence of such small vortices was also put in evidence by Brooke and Hanratty $\left[{ }^{8}\right]$, who found that each turbulenceproducing quasi-streamwise vortex in the viscous wall region is created in time in the downwash of another flow-oriented vortex. Brooke and Hanratty $\left[{ }^{8}\right]$ proposed a turbulence regeneration cycle in which each mature quasi-streamwise vortex, the parent vortex (the green vortex in Fig. 7a), produces a small quasi-streamwise vortex of opposite sign, the offspring vortex. Following other interpretations, the offspring vortices may be interpreted as the rear, wall-touching end of a counterrotating quasi-streamwise vortex farther downstream $\left[{ }^{29,40}\right]$.

It is not our object here to focus on the turbulence regeneration cycle. However, we aim at verifying the action of the offspring vortices in trapping the particles in the wall layer. From Fig. 7a it is apparent that particles, which enter the field of the offspring vortex, may not easily escape and go under the influence of the ejection, maintained by the mature vortex. We verified if the situation just described has statistical relevance and used a visual criterion to determine whether the structure dynamics shown in Fig. 7a is statistically more probable than the others. We examined 50 flow fields spaced over time in order to have a large set of uncorrelated realizations. We observed an average sample of about 50 vortices taken from the 
same instantaneous flow field realization and we found single flow-oriented mature vortices coupled with secondary counter-rotating newly-born vortices in more than $70 \%$ of the observations. Occasionally, this coupling is not present. We detected pairs of quasi-streamwise vortices with equal strength - i.e. characterizing the legs of the so-called horseshoe vortices $\left[{ }^{45,46}\right]$ - in just $25 \%$ of the observations. Our observations agree well with previous results $\left[{ }^{29,40}\right]$.

We followed the evolution in time of the structures in Fig. 7a. Figure 7b shows the same box as Fig. 7a approximately 30 wall time units later and 150 wall units downstream. Colours and symbols in Fig. 7b have the same meaning as in Fig. 7a. We followed the evolution of the newly-born vortex with a convection velocity equal to $0.5 U_{C}$, which corresponds to the $z^{+}=10$ location of the newly-born vortex. The parent vortex, previously shown, is no longer visible whereas the pocket of negative $\omega_{x}$ has grown both in length and size and has lifted from the wall. Under the cusp of the lifted low-speed streak, the green $\Omega$ isosurface indicates the presence of a patch of positive $\omega_{x}$ vorticity that will later become a third generation vortex $\left[{ }^{8}\right]$. The clockwise-rotating vortex on the left, pertaining to the influence area of the low-speed streak on the left of the figure, is not discussed in this context.

As is apparent from Fig. 7, the role of the secondary vortex is crucial in preventing particles from being entrained in the outer flow. As discussed in the previous section, energetic strongly coherent ejections correlate well with particle fluxes away from the wall. In turn, strongly coherent ejections are generated by strong mature vortices, which at the same time are associated with offspring vortices. The combined action of the newly-born vortex and the mature vortex is such as to reduce the width of the "ejection avenue", preventing a large fraction of the particles from accessing the ejection area, which is the only way for a particle to be driven away from the wall layer $\left[{ }^{15}\right]$. Thus, the newly-born vortex acts to enhance the energy level, required to carry particles in the outer flow region, and plays a primary role in reducing overall particle mixing, through which particles in the wall layer have to pass to reach the outer flow.

As mentioned previously, particles with $\left|w_{P}\right|<10^{-3}$ (empty circles) are mostly settled under the low-speed streak in a wall layer, confined between the offspring vortex and the wall. These trapped particles were pushed toward the wall by previous downsweeps but no ejection, sufficiently energetic to re-entrain them, has occurred yet. This behaviour is likely due to the above mechanism for near-wall vortex regeneration. The birth of new vortices is associated with strong spanwise motions which counteract the wall-normal pulls due to local turbulence gradients or fluid ejections and prevent some particles from being re-entrained. The overall effect is to concentrate and keep particles within the sediment layer in elongated streaks that may be viewed as low-stress regions associated with a stagnation flow.

It is important now to underline the timing at which the different events, contributing to bringing and segregating the particles in the wall layer, occur.

A Lagrangian description of the local trajectory of particles, when under the influence of the structures described above, may help to elucidate further the particle trapping mechanisms. In Fig. 8a, we show the mature vortex and the 
offspring vortex at the same time step of Fig. 7a, together with the trajectories of a number of particles. We chose several particles in interesting positions, i.e. trapped or ejected away from the wall layer, and we tracked their trajectories backward and forward. The dot-to-dot distance on a particle trajectory is $\Delta t^{+}=0.7065$ in wall time units. During this time, we can hypothesize that turbulence structures change slightly, their average life being more than 100 wall time units $\left[{ }^{8,29,47}\right]$. In this figure, we also show the instantaneous flow streamlines, calculated at the same time at which we visualized the quasi-streamwise vortices. Positive values of the stream function $\Psi$ (dashed lines) are associated with counterclockwise-rotating vortices whereas negative values of $\Psi$ (dotted lines) are associated with clockwiserotating vortices. Streamlines with $\Psi=0$ are plotted as solid lines. Consider the three particles labelled $A, B$ and $C$ in Fig. 8a (their position at the time of the figure is identified by the black dot). Tracking their trajectory backward, we observe the three particles left from the same fluid environment and, tracking their trajectory forward, we see that they end up in the same neighbourhood. However, and this is important for their future destiny, they have different curvatures of the trajectories. Going now to Fig. 8b, we can see the wall structure 2.8 wall time units later, approximately 11 wall units downstream. After this short time, the large vortex changed slightly and the smaller vortex moved farther from the wall. The position of the three particles is again identified by the black dot. We shall now consider the overall trajectory of the three particles: the particle, labelled $A$, follows a neat path around the mature quasi-streamwise vortex in green, and after being swept toward the wall, enters the outer flow driven by the ejection. The particle, labelled $B$, follows a path similar to that of the particle $A$, but before being entrained by the ejection it bounces elastically on the wall. Particle $C$ goes under the offspring vortex, very close to the wall, where it finds an adverse flow, which pushes it backward parallel to the wall. This particle will be confined longer in the viscous wall layer.

In Fig. 8c, we examined a similar situation, generated by the clockwiserotating quasi-streamwise vortex (in red), with all symbols and positioning of letters maintaining the same meaning. Again, we chose three particles with different destiny. Particle $D$, after being entrained by the sweep, is able to pass between the mature vortex and the offspring vortex, along the black streamline $\Psi=0$, which identifies the only escape way from the wall region. Particle $E$ is driven too far under the offspring vortex and is not able to escape from the wall region. Particle $F$ bounces off the wall and is able to follow the ejection to the outer flow.

If we now consider the streamline patterns in Fig. 8, the action of the offspring vortex in trapping the particles in the wall layer is evident. The flow regions, bordering the $\Psi=0$ streamlines, indicated with black arrows, are source flows from the wall region. The presence of the offspring vortex, associated with the effects of the mature vortex, contributes to squeeze these regions increasing contour density, thus reducing the probability for a particle to be entrained to the outer flow.

The aim of this Section was to elucidate the mechanisms by which inertial particles are trapped in the near-wall region by the syncronicity among the turbulent 
transfer mechanisms, namely strongly coherent sweeps and ejections, and the regeneration cycle of the quasi-streamwise vortices. To this purpose, we focused on the smaller particles in our set $\left(\tau_{P}^{+}=3.8\right)$, which show the higher tendency to respond to the trapping mechanism we are discussing. A sample analysis, conducted for larger particles, gave qualitatively similar results indicating that for the investigated particle time-scales at $R e_{\tau}=150$ the particles segregation mechanisms are similar. Quantitative results (not shown here) are different due to the different particle inertia.

If, as in $\left[{ }^{41}\right]$, we employ the term hairpin vortex to represent the broad gender of quasi-streamwise structures, which may be or may not be associated to a counterrotating parallel vortex by a head of spanwise vorticity, the mechanism we propose for particle transfer in the region very near to the wall is consistent with the framework by Robinson $\left[{ }^{3}\right]$, who observed that the quasi-streamwise hairpin vortex legs dominate the buffer layer, whereas inclined necks and heads predominate in the logarithmic layer.

Furthermore, our mechanisms for particle transfer and trapping are also consistent with other experimental observations. Niño and Garcia $\left.{ }^{[5}\right]$ observed that particle re-entrainment is most effectively performed by intense bursts of the wall fluid, occurring almost in correspondence with typical shear layers, travelling at a convection velocity of about 0.70 to 0.80 of the local mean velocity. Similar shear layers have been characterized by Adrian et al. $\left[{ }^{41}\right]$ in the frame of the hairpin-packet mechanism for turbulence regeneration cycle. The hairpin packets travel at different convection velocities, enclose a low velocity fluid region, characterized by a series of Q2 events, and also enclose internal shear layers travelling at a velocity of $0.8 U_{\infty}$, where $U_{\infty}$ is the free-stream velocity. In a recent paper, Schoppa and Hussain $\left[{ }^{48}\right]$ gave an explanation for the formation of these shear layers and observed that "where a streamwise vortex ... is formed on one flank of a streak, an internal shear layer forms on the other flank of the streak". The same authors, analysing turbulence structures in a minimal channel flow $\left[{ }^{29,47}\right]$, ascribe to the low-speed streak lateral instability the responsibility for quasi-streamwise vortices regeneration. In particular, they observed that lowspeed streaks go through quiescent phases - vortex-less low-speed streaks - and through active phases, during which the low-speed streak surface bulges outward, taking a cusp-like shape, and coherent regions of streamwise vorticity, roughly centred about 30 wall units away from the wall appear, flanking the low-speed streak (see Fig. 2 in $\left[{ }^{47}\right]$ and Fig. 10 in $\left[{ }^{38}\right]$ ). These regions of streamwise vorticity are the signature of the forward-end of quasi-streamwise vortices, which are strong enough to generate strong ejections able to re-entrain particles in the outer flow. In Fig. 7a, the low-speed streak is just going through one of such active phases and has the characteristic cusp-like shape flanked by a counter-clockwise rotating quasi-streamwise vortex which, in turn, is generating a strong ejection of the wall fluid.

Apparently, our findings support previous observations on particle re-entrainment mechanisms by proving the efficiency of the strongly coherent ejections. Yet, 
they add information, since strongly coherent ejections are extremely effective in transferring particles from the wall region to the outer flow provided that particles are not prevented to reach the region where ejections can entrain them.

To provide a unifying pictorial view of the mechanisms, discussed in this paper, by statistical means, in Fig. 9 an instantaneous snapshot of particle distribution and turbulent coherent structures in the near-wall region of the channel is shown, superposed to the 2D footprint of the wall shear stress. The perspective view and the inset top view focus on a window of the computational domain (dimensions are $180 \times 135 \times 55$ in dimensionless wall units) and give a clear rendering of the physical phenomenon. Using the $\Omega=0.03$ isosurface with both signs, we have identified two quasi-streamwise vortices; for clarity of presentation, we only show the rearend of the mature counter-clockwise-rotating vortex (blue isosurface) together with the forward-end of the secondary clockwise-rotating vortex (red isosurface). As expected, the two vortices are separated by the $\Psi=0$ streamsurface (green isosurface), cut at $z^{+}=20$. The environment, bordering the streamsurface, is an ejection-like outflow region, characterized by low values of the wall shear stress (in blue). As mentioned previously, this region is squeezed by two vortices, which act to reduce the probability for particles to be resuspended into the outer flow $\left[{ }^{9}\right]$. Most of the particles, plotted as light grey spheres, accumulate in the ejectionlike region, avoiding the high shear stress sweep-like environment at the wall (in red). It is also apparent that coherent vortices are not able to entrain particles. Events shown in Fig. 9 have been observed in a large number of instants at different locations of the channel.

\section{SUMMARY AND CONCLUSIONS}

The identification of the mechanisms, leading to particle transfer in the wall region and to particle segregation in regions of the viscous sublayer, where the streamwise fluid velocity is lower than the mean, is of fundamental significance for a number of technological and environmental applications, ranging from new gas-cleaning devices and prediction of environmental sedimentation processes to the sizing and control of surface chemical reactions. A sound understanding and characterization of these mechanisms requires analysis of the interactions between the coherent structures, controlling the turbulent transfer at the wall and particle dynamics.

We had two objects in this research. The first was to review the dynamics of boundary layer turbulence with specific reference to the convective mechanisms, which transfer particles toward the wall and toward the outer flow.

The second was to explain, from a physical viewpoint, the mechanisms for particle segregation within the boundary layer, linking particle transfer phenomena to the dynamics of the near-wall turbulent vortical structures. Evidence of particle accumulation in the near-wall region was observed previously $\left[{ }^{4}\right]$, and turbophoresis was the name to describe it. From a statistical viewpoint, turbophoresis 
is the outcome of the combined effects of particle inertia and of the wall on the distribution of turbulence moments. In practice, particles are convected down the gradients of turbulence intensity $\left[{ }^{5}\right]$. The main purpose of this work was to establish a model for particle trapping at the wall, based on the joint examination of particle dynamics together with the dynamics of the turbulence structures, populating the near wall region of the turbulent boundary layer.

From previous papers $\left[{ }^{28,29,38,40,47}\right]$ it was possible to establish that: i) lowspeed streaks are long-lived structures, ii) low-speed streaks are flanked by clockwise and counter-clockwise-rotating quasi-streamwise vortices, distributed mostly (in about $75 \%$ of the cases) as a staggered array, iii) quasi-streamwise vortices generate strongly coherent sweeps and ejections. In this work, we were able to verify that: iv) particles are driven toward the wall and toward the outer flow only by the strongly coherent sweeps and ejections. Examining in detail the dynamics of the wall structures in connection with the dynamics of the particles, entering and exiting the wall layer, we were able to appreciate fully the relevance of a secondary wall structure which was described in $\left[{ }^{8,49}\right]$. In particular, we focused on the near-wall region of the turbulent boundary layer and we examined the role of the very near to the wall, rear end of a quasi-streamwise vortex in preventing particles in the proximity of the wall to be re-entrained by the pumping action of the large, farther from the wall, forward end of the following quasi-streamwise vortex. The local flow structure produced by this couple prevents a number of the particles, entered in the wall layer, from being entrained toward the outer flow. In particular, even though the strongly coherent sweep events, required to drive particles to the wall, are associated with strongly coherent ejections, capable of driving the particles toward the outer flow, the simultaneous presence of the offspring vortex acts as to reduce the width of the "ejection channel". In practice, only particles which enter the wall-layer with specific trajectory curvature may be able to be entrained into the outer flow.

Our results confirm previous results on particle re-entrainment mechanisms and prove the efficiency of the strongly coherent ejections and sweeps as particle transfer mechanisms. However, we found evidence that strongly coherent ejections are extremely effective in transferring only those particles, which are not prevented to reach the region, where ejections can entrain them. The reasons for turbophoresis now appear clear: it is the syncronicity between the strongly coherent ejections, which are able to entrain the particles, and the presence of the very near to the wall, rear-end of a quasi-streamwise vortex which prevents equal in and out wall fluxes.

Current effort is aimed at developing a two-way coupling procedure for the simulation of fluid-particle flows to quantify turbulence modulation, induced by the dispersed phase $\left[{ }^{51}\right]$. 


\section{ACKNOWLEDGEMENTS}

I am very much indebted to my coworkers Cristian Marchioli, Andrea

Giusti and Maurizio Picciotto. Financial support from MIUR under Grant No. 2003099224-002 is gratefully acknowledged.

\section{REFERENCES}

1. Roshko, A. Structure of turbulent shear flows: a new look. AIAA Journal, 1976, 14, 1349_ 1357.

2. Hussain, F. Coherent structures - reality and myth. Phys. Fluids, 1983, 26, 2816-2838.

3. Robinson, S. K. Coherent motions in the turbulent boundary layer. Ann. Rev Fluid Mech., 1991, 23, 601-639.

4. Caporaloni, M., Tampieri, F., Trombetti, F. and Vittori, O. Transfer of particles in nonisotropic air turbulence. J. Atmos. Sci., 1975, 32, 565-568.

5. Reeks, M. W. The transport of discrete particles in inhomogeneous turbulence. J. Aerosol Sci., 1983, 14, 729-739.

6. Eaton, J. K. and Fessler, J. R. Preferential concentration of particles by turbulence. Int. J. Multiphase Flow, 1994, 20, 169-209.

7. Kaftori, D., Hetsroni, G. and Banerjee, S. Particle behavior in the turbulent boundary layer, II. Velocity and distribution profiles. Phys. Fluids, 1995, 7, 1107-1121.

8. Brooke, J. W., and Hanratty, T. J. Origin of turbulence-producing eddies in channel flow. Phys. Fluids A, 1993, 5, 1011-1022.

9. Marchioli, C. and Soldati, A. Mechanisms for particle transfer and segregation in turbulent boundary layer. J. Fluid Mech., 2002, 468, 283-315.

10. Marchioli, C., Giusti, A., Salvetti, M. V. and Soldati, A. Direct numerical simulation of particle wall transfer and deposition in upward turbulent pipe flow. Int. J. Multiphase Flow, 2003, 29, 1017-1038.

11. Narayanan, C., Lakehal, D., Botto, L. and Soldati, A. Mechanisms of particle deposition in a fully developed turbulent channel flow. Phys. Fluids A, 2003, 15, 763-775.

12. McLaughlin, J. B. Aerosol particle deposition in numerically simulated channel flow. Phys. Fluids, 1989, 1, 1211-1224.

13. Pedinotti, S., Mariotti, G. and Banerjee, S. Direct numerical simulation of particle behavior in the wall region of turbulent flow in horizontal channels. Int. J. Multiphase Flow, 1992, 18, 927-941.

14. Kaftori, D., Hetsroni, G. and Banerjee, S. Particle behavior in the turbulent boundary layer, I. Motion, deposition, and entrainment. Phys. Fluids, 1995, 7, 1095-1106.

15. Niño, Y. and Garcia, M. H. Experiments on particle-turbulence interactions in the nearwall region of an open channel flow: implications for sediment transport. J. Fluid Mech., 1996, 326, 285-319.

16. Zhang, H. and Ahmadi, G. Aerosol particle transport and deposition in vertical and horizontal turbulent duct flows. J. Fluid Mech., 2000, 406, 55-80.

17. Marchioli, C., Picciotto, M. and Soldati, A. Interactions between turbulence stucture and inertial particles in boundary layers: mechanisms for particle transfer and preferential distribution. In Modelling and Experimentation in Two-phase Flow (Bertola, V., ed.). CISM Courses and Lectures, 2004, vol. 450, 383-434, Springer.

18. Uijttewaal, W. S. J. and Oliemans, R. V. A. Particle dispersion and deposition in direct numerical and large eddy simulations of vertical pipe flows. Phys. Fluids, 1996, 8, 2590-2604.

19. Portela, L. M., Cota, P. and Oliemans, R. V. A. Numerical study of the near-wall behaviour of particles in turbulent pipe flows. Powder Technol., 2002, 125, 149-157. 
20. Cerbelli, S., Giusti, A. and Soldati, S. ADE approach to predicting dispersion of heavy particles in wall bounded turbulence. Int. J. Multiphase Flow, 2001, 27, 1861-1879.

21. Kallio, G. A. and Reeks, M. W. A numerical simulation of particle deposition in turbulent boundary layers. Int. J. Multiphase Flow, 1989, 15, 433-446.

22. Chen, M. and McLaughlin, J. B. A new correlation for the aerosol deposition rate in vertical ducts. J. Colloid Interface Sci., 1995, 169, 437-455.

23. Papavassiliou, D. V. and Hanratty, T. J. Interpretation of large structures observed in a turbulent plane Couette flow. Int. J. Heat Fluid Flow, 1997, 18, 55-69.

24. Kasagi, N. and Iida, O. Progress in direct numerical simulation of turbulent heat transfer. In 5th ASME/JSME Joint Thermal Engineering Conference. San Diego, 1999, CDROM.

25. De Angelis, V., Lombardi, P., Andreussi, P. and Banerjee, S. Microphysics of scalar transfer at air-water interfaces. In IMA Conference on Wind over Wave Couplings. Salford, 1997, Oxford University Press, 1997.

26. Kline, S. J., Reynolds, W. C., Schraub, F. A. and Runstadler, P. W. The structure of turbulent boundary layer. J. Fluid Mech., 1967, 70, 741-773.

27. Kline, S. J. and Robinson, S. K. Quasi-coherent structures in the turbulent boundary layer. Part 1. Status report on community-wide survey of the data. In Near-Wall Turbulence (Kline, S. J. and Afgan, N. H., eds.). Hemisphere, New York, 1990, 218-247.

28. Jeong, J., Hussain, F., Schoppa, W. and Kim, J. Coherent structures near the wall in a turbulent channel flow. J. Fluid Mech., 1997, 332, 185-214.

29. Schoppa, W. and Hussain, F. Genesis and dynamics of coherent structures in near-wall turbulence. In Self-sustaining Mechanisms of Wall Turbulence (Panton, R., ed.). Advances in Fluid Mechanics. Computational Mechanics Publications, Southampton, 1997, vol. 15, 385-422.

30. Jimenez, J. and Pinelli, A. The autonomous cycle of near-wall turbulence. J. Fluid Mech., 1999, 389, 335-359.

31. Bonnet, J. P., Delville, J., Glauser, M. N., Antonia, R. A., Bisset, D. K., Cole, D. R., Fiedler, H. E., Garem, J. H., Hilberg, D., Jeong, J. et al. Collaborative testing of eddy structure identification methods in free turbulent shear flows. Exp. Fluids, 1998, 25, $197-225$.

32. Hunt, J. C. R., Wray, A. A. and Moin, P. Eddies, stream and covergence zones in turbulent flows. Center of Turbulence Research Rep., Stanford, CTR-S88, 1998, 193.

33. Dubief, Y. and Delcayre, F. On coherent-vortex identification in turbulence. J. Turbulence, $2000,1,11-32$.

34. Perry, A. and Chong, M. S. A description of eddying motions and flow patterns using critical point concepts. Annu. Rev. Fluid Mech., 1987, 9, 125-148.

35. Chong, M. S., Perry, A. and Cantwell, B. J. A general classification of three-dimensional flow fields. Phys. Fluids A, 1990, 2, 765-777.

36. Jeong, J. and Hussain, F. On the identification of a vortex. J. Fluid Mech., 1995, 285, 69-83.

37. Lombardi, P., De Angelis, V. and Banerjee, S. Direct numerical simulation of nearinterface turbulence in coupled gas-liquid flow. Phys. Fluids, 1996, 8, 1643-1665.

38. Soldati, A. and Marchioli, C. Prospects for modulation of turbulent boundary layer by EHD flows. In Turbulence Structure and Modulation (Soldati, A. and Monti, R., eds.). CISM Courses and Lectures, 2001, vol. 415, 119-160, Springer.

39. Picciotto, M., Marchioli, C. and Soldati, A. Remarks on the distribution of inertial particles in the wall region of a turbulent boundary layer. Forthcoming.

40. Schoppa, W. and Hussain, F. New aspects of vortex dynamics relevant to coherent structures in turbulent flows. In Eddy Structure Identification (Bonnet, J. P., ed.). CISM Courses and Lectures, 1996, vol. 353, 61-143, Springer. 
41. Adrian, R. J., Meinhart, C. D. and Tomkins, C. D. Vortex organization in the outer region of the turbulent boundary layer. J. Fluid Mech., 2000, 422, 1-54.

42. Guezennec, Y. G., Piomelli, U. and Kim, J. On the shape and dynamics of wall structures in turbulent channel flow. Phys. Fluids A, 1989, 1, 764-766.

43. Lyons, S. L., Hanratty, T. J. and McLaughlin, J. B. Large computer simulation of fully developed turbulent channel flow with heat transfer. Int. J. Numer. Meth. Fluids, 1991, 13, 999-1028.

44. Kim, J., Moin, P. and Moser, R. Turbulence statistics in fully developed channel flow at low Reynolds number. J. Fluid Mech., 1987, 177, 133-166.

45. Zhou, J., Adrian, R. J., Balachandar, S. and Kendall, T. M. Mechanisms for generating coherent packets of hairpin vortices in channel flow. J. Fluid Mech., 1999, 387, 353396.

46. Choi, K.-S. Turbulent drag reduction mechanisms: strategies for turbulence management. In Turbulence Modulation and Control (Soldati, A. and Monti, R., eds.). CISM Courses and Lectures, 2001, vol. 415, 161-211, Springer.

47. Schoppa, W. and Hussain, F. Coherent structure dynamics in near-wall turbulence. J. Fluid Dynamics Res., 2000, 26, 119-139.

48. Schoppa, W. and Hussain, F. Coherent structure generation in near-wall turbulence. $J$. Fluid Mech., 2002, 453, 57-108.

49. Bernard, P. S., Thomas, J. M. and Handler, R. A. Vortex dynamics and the production of Reynolds stress. J. Fluid Mech., 1993, 253, 385-419.

50. Giusti, A., Nonino, C. and Soldati, A. Finite element simulation of fluid-particle interaction in lid-driven cavity flow. In Proc. 21st U.I.T. Conference (Cortella, G. and Croce, G., eds.). Udine, 2003, 453-460.

\title{
Inertsete mikroosakeste füüsikalised ülekandeprotsessid turbulentses piirkihis
}

\begin{abstract}
Alfredo Soldati
On kirjeldatud inertsete tahkete osakeste jaotumist turbulentses vooluses ja nende väljasettimise mehhanismi. Samuti on selgitatud, et mõnedel piirkihi juhtudel leiab aset osakeste akumuleerumine seinte ääres. Arvmodelleerimise ja eksperimentaalsete tulemuste põhjal on näidatud, et vedeliku liikumine turbulentses piirkihis on vahelduv ning selle rangelt organiseeritud ja koherentset olemust väljendab suuremastaabiline liikumine. Need kvaasideterministlikud liikumised määravad dispergeerunud osakeste transpordi disperssetes voolustes.
\end{abstract}

